\title{
$\beta$-Catenin as a Tension Transmitter Revealed by AFM Nanomechanical Testing
}

\author{
$\operatorname{AUTHOR}(\mathrm{S})$ :
}

Maki, Koichiro; Han, Sung Woong; Adachi, Taiji

\section{CITATION:}

Maki, Koichiro ... [et al]. B-Catenin as a Tension Transmitter Revealed by AFM Nanomechanical Testing. Cellular and Molecular Bioengineering 2015, 8(1): 14-21

ISSUE DATE:

2015-03

URL:

http://hdl.handle.net/2433/235624

\section{RIGHT:}

This is a post-peer-review, pre-copyedit version of an article published in Cellular and Molecular Bioengineering. The final authenticated version is available online at: http://dx.doi.org/10.1007/s12195-014-0367-2.; この論文は出版社版て ありません。引用の際には出版社版をご確認ご利用ください。;This is not the published version. Please cite only the published version. 


\section{$\beta$-Catenin as a tension transmitter revealed by AFM nanomechanical}

\section{testing}

Koichiro Maki ${ }^{1,2)}$, Sung-Woong Han ${ }^{1,2)}$, Taiji Adachi ${ }^{1,2, ~ *)}$

${ }^{1}$ Department of Biomechanics, Institute for Frontier Medical Sciences, Kyoto University

53 Shogoin-Kawahara-cho, Sakyo, Kyoto 606-8507, Japan

${ }^{2}$ Department of Micro Engineering, Graduate School of Engineering, Kyoto University

Yoshida Honmachi, Sakyo, Kyoto 606-8501, Japan

An abbreviated title: $\quad \beta$-Catenin as a tension transmitter revealed by AFM

nanomechanical testing

*Corresponding Author: Taiji Adachi, Ph.D.

Mailing Address: $\quad$ Department of Biomechanics

Research Center for Nano Medical Engineering

Institute for Frontier Medical Sciences

Kyoto University

53 Shogoin-Kawahara-cho, Sakyo, Kyoto 606-8507, Japan

Telephone \& Fax: $\quad$ +81 (75) 751-4853

E-mail:

adachi@frontier.kyoto-u.ac.jp

Submitted to: $\quad$ Cellular and Molecular Bioengineering

Revised on Nov 12, 2014 


\section{Abstract}

$\beta$-Catenin, a component of adherens junction (AJ), transmits forces between cells and stabilizes their balance, thereby contributing to AJ maturation. A previous study based on a single-molecule experiment has suggested that $\beta$-catenin unfolds under tension with low mechanical stability, while another study showed its mechanical behavior bearing to high forces. Such discrepancy in mechanical behaviors of $\beta$-catenin could be a result of experimental conditions such as solvent compounds, $\mathrm{pH}$, and temperature. In the present study, we successfully tested the mechanical behaviors of $\beta$-catenin in a simple experimental condition by employing atomic force microscopy (AFM). To perform nanomechanical testing in phosphate-buffered saline (PBS) buffer without protein aggregation, we directly modified the AFM probe with $\beta$-catenin in a low concentration. We performed two types of experiments, in which a part and a whole structure of $\beta$-catenin were loaded under tension. The results of the testing indicated that $\beta$-catenin behaves as a nonlinear elastic material under tension, i.e., a mechanical component with low stiffness under low tension and high stiffness under high tension. The nonlinear elastic behavior exhibited in our experiment suggests a mechanical function of $\beta$-catenin to serve as a tension transmitter at AJs: 1 ) preserving links between AJ molecules under low tension and 2) transmitting high tension with high mechanical stability.

Key terms: $\beta$-Catenin, Nanomechanical testing, Atomic force microscopy, Mechanotransduction, Single molecule, Molecular biomechanics 


\section{Introduction}

In many biological processes, such as morphogenesis, maintenance of tissue integrity, and wound healing, cells in multicellular tissue sense and adapt to their mechanical environment, resulting in dynamic cell rearrangement and tissue deformation ${ }^{23,28}$. These processes involve mechanotransduction, in which a mechanical stimulus is converted into biochemical signals ${ }^{1 \text {, }}$ ${ }^{8,12}$. In particular, mechanotransduction contributes to intercellular interactions between cells ${ }^{4}$, 15, 35 because the force generated by the intracellular actomyosin contraction is mutually transmitted between adjacent cells via adherens junctions (AJs). Therefore, the intercellular force transmission is essential for multicellular tissue dynamics.

AJs form a link between cytoskeletal actin filaments in adjacent cells and transmit the intercellular force ${ }^{7,31}$. Component molecules, such as cadherin (a transmembrane protein involved with cell-cell adhesion), $\beta$-catenin, $\alpha$-catenin (a cytoplasmic protein that plays a role as a mechanosensor in maturation of $\mathrm{AJs}^{38}$ ), and $\mathrm{EPLIN}^{2}$, directly bind together serially in this order to interact with the force-generating actin cytoskeleton. The intercellular tension frequently fluctuates depending on the cell arrangement within the tissue and myosin activities within individual cells. Therefore, AJs and their component molecules are frequently exposed to high tension between cells ${ }^{21,32}$. To control unbalanced forces, $\alpha$-catenin at AJs adaptively matures ${ }^{38}$, and during this process, mechanical behaviors of AJ components would be a key factor in determining the characteristics of this mechano-feedback mechanism ${ }^{3,13}$. 
cadherin and $\alpha$-catenin and contains armadillo (ARM) repeats in its central region (138-664 amino acids) forming a superhelical spiral structure ${ }^{10,36}$. Such unique spiral structures play a role as tension transmitters and are found in Ankyrin (ANK) repeats with a similar superhelical spiral protein in hair cells, exhibiting low stiffness under low tension and high stiffness under high tension for mechanotransduction ${ }^{16}$. A previous single-molecule experiment showed a high mechanical stability of $\beta$-catenin when subjected to a high force ${ }^{26}$, while the other experiment suggested that $\beta$-catenin unfolds under tension with low mechanical stability ${ }^{33}$. Therefore, we considered that it is possible that the mechanical behaviors of $\beta$-catenin vary in experimental conditions such as solvent compounds, $\mathrm{pH}$, and temperature.

In the present study, we aimed to test the mechanical behaviors of $\beta$-catenin in a simple experimental condition in phosphate-buffered saline (PBS) buffer without any additional compounds. To investigate the mechanical behaviors of $\beta$-catenin under tension at the molecular level, we employed atomic force microscopy (AFM) nanomechanical testing by directly loading single molecules ${ }^{25}$. We adopted a similar rate of loading as that used in previous studies ${ }^{26,33}$ for comparison, although the rate would not necessarily match up with the in vivo condition. To test the mechanical behaviors of $\beta$-catenin considering the mechanical conditions at AJs, we performed two types of experiments, in which a part and a whole structure of $\beta$-catenin were loaded under tension. To apply the force to a part of $\beta$ catenin, single-tagged (N-terminus GST-tagged) $\beta$-catenin was introduced to experiments, 
where N-terminus GST-tag and solvent-exposed amine residues widely dispersed in ARM repeats were targeted. In addition, to load the full component of $\beta$-catenin by both its ends, double-tagged [N-terminus GST-tagged and C-terminus His-tagged] $\beta$-catenin was introduced.

\section{Materials and methods}

\subsection{Materials}

The N-terminal domain of $\beta$-catenin directly binds to $\alpha$-catenin, and the central ARM repeats interact with the cytoplasmic domain of cadherin, as shown in Fig. 1a. To analyze the mechanical behavior of $\beta$-catenin as a tension transmitter at AJs, we used two types of $\beta$ catenin fusion proteins, i.e., single-tagged and double-tagged human full-length $\beta$-catenin (Figs.1b and 1c). Single-tagged $\beta$-catenin (Fig. 1b), purchased from Abnova Corporation, was used in the AFM nanomechanical testing, where the GST tag and the solvent-exposed lysine residues were targeted (left, Fig. 2). Using single-tagged $\beta$-catenin, we targeted 26 lysines in the whole structure, of which 20 lysines were widely dispersed in ARM repeats. In addition, double-tagged $\beta$-catenin (Fig. 1c) was used in the AFM nanomechanical testing, where the GST and His tags at both termini were targeted for modification processes of the AFM tip and the substrate (right, Fig. 2). The plasmid DNA of double-tagged $\beta$-catenin was cloned into pGex6pl-7his and transformed into E-coli BL21-D3-pLysS. Double-tagged $\beta$-catenin was expressed overnight using $0.1 \mathrm{mM}$ isopropyl $\beta$-D-1-thiogalactopyranoside (IPTG) induction 
at $20^{\circ} \mathrm{C}$ and purified with a GST bulk kit (GE Healthcare Biosciences). Using double-tagged

$\beta$-catenin, we were able to load full-length $\beta$-catenin including, all 12 ARM repeats.

Surface preparation agents, i.e., (3-aminopropyl) triethoxysilane (APTES; Sigma-Aldrich Co., LLC) ${ }^{19}$ and (3-mercaptopropyl)-trimethoxy-silane (MPTMS; Sigma-Aldrich Co., LLC) were used to modify the silicon nitride AFM tip (OMCL-TR400PSA-1, Olympus Co.; spring constant $(k), 0.02 \mathrm{~N} / \mathrm{m})$ and the glass substrate. N-hydroxylsuccinimide-polyethylene glycolN-hydroxylsuccinimide (NHS-PEG-NHS ester, NANOCS Inc.; M.W., 3.4 kDa) ${ }^{18}$, maleimidepolyethylene glycol-N-hydroxylsuccinimide (Mal-PEG-NHS ester, NANOCS Inc.; M.W., 3.4 $\mathrm{kDa})^{27}$ and N -(6-maleimidocaproyloxy)sulfosuccinimide (Sulfo-EMCS, DOJINDO Laboratory) were used for the chemical modification of the AFM tip and the glass substrate. Ethanethiol (Sigma-Aldrich Co., LLC) was used for blocking the AFM tip.

\subsection{Modification of the AFM tip and the glass substrate}

The silicon nitride AFM cantilever (spring constant, $0.02 \mathrm{~N} / \mathrm{m}$ ) was used for measuring piconewton order small forces ${ }^{24}$. The AFM tip was washed with Milli-Q water (EMD Millipore Co.), oxidized using ozone cleaner, and treated with 2\% APTES in 99.5\% ethanol for $15 \mathrm{~min}$. It was then treated with $5 \mathrm{mM}$ NHS-PEG-Mal ester for $30 \mathrm{~min}$ and with $10 \mathrm{mM}$ glutathione for 1 h. $\beta$-Catenin $(1 \mu \mathrm{g} / \mathrm{mL})$ was modified on the AFM tip using glutathione-GST affinity binding ${ }^{5}$ for 2 h. Finally, the unreacted maleimide groups were blocked using $0.1 \%$ ethanethiol in phosphate-buffered saline (PBS) for $5 \mathrm{~min}$. All reactions were performed at 
$27^{\circ} \mathrm{C}$.

To modify the substrate, a cover glass was washed with Milli-Q water, oxidized, and treated with 2\% MPTMS in 99.5\% ethanol for 15 min. In double-tagged $\beta$-catenin experiments, the substrate was treated with 0.4 mM maleimide- $\mathrm{C}_{3}-\mathrm{NTA}$ (Mal- $\mathrm{C}_{3}-\mathrm{NTA}$; DOJINDO Lab.) for $1 \mathrm{~h}$, with $10 \mathrm{mM} \mathrm{NiCl}$ (Wako Pure Chemical Industries) for $30 \mathrm{~min}$, followed by washing with PBS. In single-tagged $\beta$-catenin experiments, the APTES glass substrate was treated with 5 mM NHS-PEG-NHS for 30 min and washed with PBS.

In our experimental settings as shown in Fig. 2, there could be nonspecific linkages between 1) the AFM tip and the substrate and 2) the PEG linker and the substrate. To distinguish the force responses of $\beta$-catenin with nonspecific linkages, we performed control experiments 1) without $\beta$-catenin and 2) without PEG linker. For experiments without $\beta$ catenin, we modified the AFM tip and the substrate in the same process, except without $\beta$ catenin. For the experiment without PEG linker, we used $1 \mathrm{mM}$ Sulfo-EMCS instead of MalPEG-NHS for modifying the AFM tip.

\section{3. $\beta$-Catenin single-molecule force spectroscopy}

A Nanowizard III BioAFM (JPK Instruments, Berlin, Germany) was used for the $\beta$ catenin nanomechanical testing. The spring constant of each AFM cantilever was calibrated using the energy equipartition theorem. The AFM nanomechanical testing of $\beta$-catenin was conducted in PBS (pH 7.4). The double-tagged $\beta$-catenin-modified AFM tip was approached 
at a speed of $0.2 \mu \mathrm{m} / \mathrm{s}$ and it was in contact with the glass substrate for $5 \mathrm{~s}$ to facilitate the binding of the C-terminus histidine tag of $\beta$-catenin and the substrate $\mathrm{Ni}^{2+}$ nitrilotriacetic acid $\left(\mathrm{Ni}^{2+}\right.$-NTA). The single-tagged $\beta$-catenin-modified AFM tip was approached in the same manner to facilitate binding of the external amino group of $\beta$-catenin with the NHS ester. We targeted 20 lysine residues, which were exposed to the solvent in ARM repeats ${ }^{36}$ (Fig. 1b) and applied tensile force to various numbers of ARM-repeats. The tip was then retracted at the same speed. To verify the elasticity of $\beta$-catenin under low tension, we performed a loadingunloading experiment. The retraction of $\beta$-catenin from the substrate was prevented by a gradual reduction of the piezo-moving distance $(d)$ to 50 , 30, and $20 \mathrm{~nm}$ until the appearance of the unloading curve, taking into account the retract extension observed from results in the loading experiment. Force $(F)$ versus $d$ curves were obtained and converted into $F$ versus $\beta$ catenin extension $\Delta L$ curves, referred to as the force curves, by subtracting the cantilever deflection from $d$.

\section{4. $\beta$-Catenin spring constants analysis}

To quantify the mechanical properties of $\beta$-catenin under tension, we estimated the $\beta$ catenin spring constants $k$ from the force curves. The spring constant $k$ was determined as the average value of the tangent stiffness] $[d F / d(\Delta L)]$ (first-order derivatives of the force curve) in the specified linear region, as described below. Initially, the base line of the applied force was determined as the average value of force points after unbinding, and the base line of 
extension was determined where the smoothed force is $0 \mathrm{pN}$. Next the first- and second-order derivatives of the force curves $\left[d F / d(\Delta L)\right.$ and $\left.d^{2} F / d(\Delta L)^{2}\right]$, respectively, were calculated. In the derivation process, each curve was smoothed using a simple moving average, where the number of datum points for the average was determined as 25 before and after the averaging point. Subsequently, the linear region was specified, where the second order derivatives take values within the determined range of $-1<d^{2} F / d(\Delta L)^{2}<1$. All parameters for calculation, such as the data points for the moving average and the second order derivatives range to determine linear regions, were set based on the correlation coefficient $\left(R^{2}>0.95\right)$ between the force and linear regression curves. Finally, we calculated the spring constants $k$ as the average of the first-order differentiated force in the linear region.

\subsection{Statistical analysis}

To confirm that the spring constants $k$ of single-tagged and double-tagged $\beta$-catenins have different values, we used an $F$ test, which verifies the hypothesis that two distributions have the same standard deviations $\sigma_{1}$ and $\sigma_{2}$. The spring constants of single-tagged ( $\left.n=66\right)$ and double-tagged $(n=34) \beta$-catenins were compared by the $F$ test with p-value of 0.001 .

\subsection{Polymer chain models}

In our study, we analyzed the mechanical behaviors of $\beta$-catenin under high tension by fitting three polymer chain models to force curves: 1) freely jointed chain (FJC) model $^{29}$ 2) 
worm-like chain (WLC) model $^{20}$, and 3) two-state WLC model ${ }^{30}$.

The FJC model describes the relationship between the applied force $F$ and extension $\Delta L$

expressing the entropy elasticity as

$$
\Delta L=L\left[\operatorname{coth}\left(\frac{F \cdot b}{k_{\mathrm{B}} T}\right)-\frac{k_{\mathrm{B}} T}{F \cdot b}\right]
$$

where $k_{\mathrm{B}}$ is Boltzmann constant, $T$ is temperature, $b$ is the Kuhn length and $L$ is the fully extended length.

The WLC model also describes the relationship between force and extension considering the bending energy of polymer chain as

$$
F=\frac{k_{\mathrm{B}} T}{p}\left[\frac{1}{4}\left(1-\frac{\Delta L}{L_{\mathrm{c}}}\right)^{-2}+\frac{\Delta L}{L_{\mathrm{c}}}-\frac{1}{4}\right]
$$

where $p$ is the persistent length and $L_{\mathrm{c}}$ is the contour length.

Two-state WLC model is an advanced model of WLC considering the conformational transition of local structures from initial state to the other state. The force $F$ is given by

$$
F=\frac{k_{\mathrm{B}} T}{p}\left[\frac{1}{4}\left(1-\frac{\Delta L}{L_{\mathrm{c}}{ }^{\prime}}\right)^{-2}+\frac{\Delta L}{L_{\mathrm{c}}{ }^{\prime}}-\frac{1}{4}\right],
$$

where $L_{\mathrm{c}}{ }^{\prime}$ is the contour length of two-state WLC model which extends under force $F$. The contour length $L_{\mathrm{c}}{ }^{\prime}$ is given by

$$
L_{\mathrm{c}}^{\prime}=L_{\mathrm{c} 0}+n \cdot \Delta L_{\mathrm{s}} \cdot \gamma(F)
$$

where $L_{\mathrm{c} 0}$ is the contour length at initial state, $n$ is the number of local structures, $\Delta L_{\mathrm{s}}$ is the increase in extension for each local structure, and $\gamma(F)$ is the possibility of conformational transition of local structure that is given by 


$$
\gamma(F)=\left[1+\exp \left(\frac{\Delta G_{\mathrm{s}}-F \cdot \Delta L_{\mathrm{s}}}{k_{\mathrm{B}} T}\right)\right]^{-1}
$$

where $\Delta G_{\mathrm{s}}$ is the free-energy change in conformational transition. The transition force $F_{\mathrm{t}}$ and increase in contour length $\Delta L_{\mathrm{c}}$ are given by $\Delta G_{\mathrm{s}} / \Delta L_{\mathrm{s}}$ and $n \cdot \Delta L_{\mathrm{s}}$, respectively. Force curves obtained in our experiments for single-tagged $\beta$-catenin were fitted by these three polymer chain models in least-square analysis.

\section{Results}

Figures 3a and 3b show the typical force curves obtained in the nanomechanical testing of $\beta$-catenin using AFM. Both single-tagged (green lines, Fig. 3a) and double-tagged (orange lines, Fig. 3b) $\beta$-catenin exhibited nonlinear behaviors during the loading process, showing distinct linear regions under low tension, as shown in Fig. 3c. Nonspecific tethers with small rupture force $F_{\mathrm{R}}(<50 \mathrm{pN})$ were observed in the experiment without $\beta$-catenin, whereas specific tethers $\left(F_{\mathrm{R}}>100 \mathrm{pN}\right)$ were observed in the experiment without a PEG linker. These results indicated that our results were caused by $\beta$-catenin behaviors. Unlike double-tagged $\beta$ catenin (Fig. 3b), single-tagged $\beta$-catenin (Fig. 3a) exhibited various spring constants $k$ in linear regions caused by the variation of reacted amine residues of $\beta$-catenin. Under high tension, $\beta$-catenin behaved as a rather rigid material and was not fully unfolded, which was confirmed by the force curves without any tension relaxation before unbinding of the tip from the substrate and by the fact that maximum extensions were much shorter than the estimated extended full length of $\beta$-catenin (310 nm). In addition, nonlinear behavior was observed in 
the loading-unloading experiments using both single-tagged and double-tagged $\beta$-catenin, in which the loading (orange line) and unloading (blue line) curves were fully reversible, as shown in Fig. 3d. These results suggest that $\beta$-catenin plays a role as a nonlinear elastic material under tension.

To confirm that single $\beta$-catenin molecules were loaded, we evaluated the rupture force $\left(F_{\mathrm{R}}\right)$, which was the peak value of $F$ in the force curves, as shown in Fig. 3c. The histogram of $F_{\mathrm{R}}$ in the single-tagged $\beta$-catenin experiment (green bar in Fig. 3e) exhibited two peaks ${ }^{6,22}$. We fitted the histogram with a weighted mixture Gaussian distribution, where the number of distributions was determined so as to minimize the residual sum of squares with the condition that each constitutive distribution has a weight larger than $10 \%$. The two peaks of the histogram indicated that the force curves were caused by single molecule (506 \pm 163 (Mean \pm S.D.) $\mathrm{pN}$ ) and double molecule $(901 \pm 136 \mathrm{pN}) \beta$-catenin. The double-molecule force curves with high rupture force did not show significant increases in extension compared to singlemolecule force curves, because they were bound to the substrate in a parallel way. In contrast, the histogram depicting $F_{\mathrm{R}}$ in the double-tagged $\beta$-catenin experiment (orange bar in Fig. 3e) exhibited a single peak representing the unbinding of a single NTA-Ni ${ }^{2+}$-His bond, whereas a previous study suggested that the histogram exhibited three maximal peaks corresponding to single, double, and triple bonds because the His 6 -tag contains up to three binding sites for NTA-Ni ${ }^{2+} 34$. In our results, the force curves would reflect the mechanical behaviors of single molecule double-tagged $\beta$-catenin. 
To quantify the mechanical properties of $\beta$-catenin, spring constants $k$ in linear regions of the force curves were calculated (Fig. 3c). The force curves of single-tagged $\beta$-catenin were extracted, and the value of $F_{\mathrm{R}}$ was found to be $506 \pm 192 \mathrm{pN}$ (half width at half maximum of first peak) to evaluate the mechanical properties of single molecules. Spring constant $k$ of the linear region was found to be $6.5 \pm 3.0 \mathrm{pN} / \mathrm{nm}$ for single-tagged $\beta$-catenin and rather smaller values $3.9 \pm 1.3 \mathrm{pN} / \mathrm{nm}$ for double-tagged $\beta$-catenin. The dispersion for single-tagged $\beta$ catenin ( $n=66)$ was significantly larger than the corresponding dispersion for double-tagged $\beta$-catenin $(n=34)[F$ test $(P<0.001)]$, which was caused from the variation in reacted amines of single-tagged $\beta$-catenin, in other words, variation in the number of ARM repeats directly loaded. This result indicates that the spring constant $k$ of $\beta$-catenin decreases according to the increasing number of ARM repeats. Such a property as a "nanospring” has been reported by Lee et al. (2006) on ANK repeats that form the same superhelical structure as ARM repeats. For analyzing the $\beta$-catenin behaviors under high tension, we fitted polymer chain models to the force curves of single-tagged $\beta$-catenin (Fig. 3f). Force curves (green line) were better explained by the simple WLC model (purple long-dashed line) than the FJC model (blue short-dashed line). In addition, we found that the two-state WLC model (red solid line), which considers the increase in the contour length caused by conformational transition from the initial state to the other state, showed the best fits to the force curves. The conformational transition from the initial state (red dotted line) occurred at high tension $\left(F_{\mathrm{t}}>150 \mathrm{pN}\right)$, whereas the increase in the contour length, $\Delta L_{\mathrm{c}}$, was 1-2 nm (from red dashed line to solid 
line, Fig. 3f). These results indicated that $\beta$-catenin underwent minor conformational transition to more rigid state under high tension. The extended lengths in our experiments ( $25 \mathrm{~nm}$ ) were significantly shorter than the expected fully extended length ( 304 nm), considering that the contour length of each amino acid is $0.4 \mathrm{~nm}$. Our result indicated that $\beta$ catenin undergoes minor conformational transition and is not fully unfolded with high mechanical stability.

\section{Discussion}

Our experiments using AFM revealed the mechanical behaviors of $\beta$-catenin under tension at the molecular level. $\beta$-Catenin behaved as a nonlinear elastic material under tension with a linear response under low tension and a rather rigid response under high tension. In addition, such a nonlinear elastic behavior of $\beta$-catenin would correspond to the in vivo situation because the tension applied to $\beta$-catenin in our experiments is in the same range as the tension exerted on single actin filaments ${ }^{11}$. We accordingly suggest that $\beta$-catenin functions as a flexible component that preserves links between AJ molecules under low tension, though acting as a rigid tension transmitter with high mechanical stability under high tension. Thus, $\beta$-catenin would contribute to regulating the intercellular force balance as a tension transmitter.

Here, we discuss the mechanical contribution of non-ARM domains to the function of $\beta$ catenin as a tension transmitter. The $\mathrm{N}$-terminal domain (1-137 amino acids) of $\beta$-catenin 
directly binds to $\alpha$-catenin, and the C-terminal domain (665-781 amino acids) does not interact with cadherin. Because both $\mathrm{N}$ - and C-terminal regions are unable to form stable structure by themselves $^{9,10}$, it is assumed that the non-ARM domains make no contribution in increasing the tension during the loading process. Moreover, in experiments using doubletagged $\beta$-catenin, we infrequently obtained the force curves that showed a certain extension without an increase in tension, caused by unstable non-ARM domains before load-bearing region with nonlinear behaviors. This result suggested that non-ARM domains exhibit extension, but do not play a significant mechanical role of $\beta$-catenin as a tension transmitter.

In our study, the nanomechanical testing was performed for isolated $\beta$-catenin fusion proteins under slightly different conditions from physiological ones. For example, a previous report by Valbuena ${ }^{33}$ suggested that the interaction between $\beta$-catenin and cadherin is essential for $\beta$-catenin to transmit intercellular tension with high mechanical stability. To examine the mechanical stability of $\beta$-catenin itself, we performed experiments for single-tagged $\beta$-catenin in $8 \mathrm{M}$ urea-PBS, a protein solubilizing agent. The resulting force curves showed linear regions under low tension and rigid regions under high tension without tension relaxation, which corresponded well with the curves obtained in experiments without urea. This result is in agreement with the report ${ }^{9}$ that showed ARM repeats structure of $\beta$-catenin conserved in 2.4 M urea. Furthermore, the report (uploaded to the Protein Data Bank under the PDB code 4EVT) showed that $\beta$-catenin treated by $8.3 \mathrm{M}$ urea retains its ARM repeats structure. Therefore, experiments in urea confirmed the high mechanical stability of $\beta$-catenin. 
The force range of molecular behaviors remains to be discussed. For the main results, the experiment was performed with a loading rate of $4000 \mathrm{pN} / \mathrm{s}$. We also performed the experiments at a lower loading rate of $1000 \mathrm{pN} / \mathrm{s}$, a rate similar to that in Valbuena's experiments, but unfolding events were not observed. Although we could not determine the exact reasons for the discrepancy in the results with the report, it could be because of the differences in the solvent compounds and testing temperature. The solvent compounds could affect the mechanical behaviors of proteins ${ }^{17}$. In Valbuena's experiments, PBS buffer containing $5 \mathrm{mM}$ dithiothreitol (DTT), which avoids aggregation, was used for 0.3-0.4 $\mathrm{mg} / \mathrm{mL} \beta$-catenin. In contrast, we used PBS buffer without any additional compounds for a lower concentration ( $1 \mu \mathrm{g} / \mathrm{mL})$ of $\beta$-catenin. In addition, the testing temperature could affect the helix content of protein and its unfolding force ${ }^{14}$. Nevertheless, it is possible that there are undiscovered molecular mechanisms that clearly explain the discrepancy in the results. For example, a previous report ${ }^{26}$ suggested that $\beta$-catenin has various conformations in equilibrium. Obviously, other experimental methods that enable a low-loading-rate experiment, such as magnetic tweezers ${ }^{37}$, are required to estimate the molecular behaviors under physiological conditions. In our study, we report one aspect of the function of $\beta$-catenin as a tension transmitter at AJs.

In the present study, we showed the mechanical behaviors of $\beta$-catenin as a tension transmitter at intercellular AJs, employing the method of AFM nanomechanical testing. This direct measurement of molecular behavior can offer new insights into force-induced 
dynamical mechanisms in multicellular systems. In future work, we will try to achieve an overall picture of molecular mechanotransduction mechanisms at AJs by analyzing the mechanical behaviors of other constituent molecules such as cadherin and $\alpha$-catenin, employing nanomechanical testing.

\section{Acknowledgments}

We thank Piotr E. Marszalek and Minkyu Kim for the plasmid DNA of N-Terminus GST tagged and C-terminus His tagged (Double-tagged) human full-length $\beta$-catenin. This work was partly supported by the Funding Program for Next Generation World-Leading Researchers (NEXT Program: LR017) and the Platform for Dynamic Approaches to Living System from the MEXT, Japan and by the SEI Group CSR Foundation, Japan. Koichiro Maki was supported by the JSPS Research Fellowships for Young Scientists from the Japan Society for the Promotion of Science.

\section{Conflict of Interest}

Koichiro Maki, Sung-Woong Han, and Taiji Adachi declare that they have no conflict of interest.

\section{Ethical Standards}

No human or animal studies were carried out by the authors for this article. 


\section{References}

1. Adachi, T., Kameo, Y., and Hojo, M. Trabecular bone remodelling simulation considering osteocytic response to fluid-induced shear stress. Philosophical transactions. Series A, Mathematical, physical, and engineering sciences 368:2669-2682, 2010.

2. Chervin-Pétinot, A., Courçon, M., Almagro, S., Nicolas, A., Grichine, A., Grunwald, D., Prandini, M.-H., Huber, P., Gulino-Debrac, D. Epithelial protein lost in neoplasm (EPLIN) interacts with $\alpha$-catenin and actin filaments in endothelial cells and stabilizes vascular capillary network in vitro. The Journal of biological chemistry 287:7556-7572, 2012.

3. Choi, H.-J., Pokutta, S., Cadwell, G. W., Bobkov, A. A, Bankston, L. A, Liddington, R. C., and Weis, W. I. $\alpha$ E-catenin is an autoinhibited molecule that coactivates vinculin. Proceedings of the National Academy of Sciences of the United States of America 109:8576-8581, 2012.

4. DuFort, C. C., Paszek, M. J., and Weaver, V. M. Balancing forces: architectural control of mechanotransduction. Nature reviews. Molecular cell biology 12:308-319, 2011.

5. Habig, H. W., Pabst, J. M., and Jakoby, B. W. Glutathione S-Transferase: The first enzymatic step in mercapturic acid formation, The journal of biological chemistry 249:7130-7139, 1974.

6. Han, S.-W., Mieda, S., Nakamura, C., Kihara, T., Nakamura, N., and Miyake, J. Successive detection of insulin-like growth factor-II bound to receptors on a living cell surface using an AFM. Journal of molecular recognition 24:17-22, 2011.

7. Harris, T. J. C., and Tepass, U. Adherens junctions: from molecules to morphogenesis. Nature reviews. Molecular cell biology 11:502-514, 2010.

8. Hoffman, B. D., Grashoff, C., and Schwartz, M. A. Dynamic molecular processes mediate cellular mechanotransduction. Nature 475:316-323, 2011.

9. Huber, A. H., Nelson, W. J., and Weis, W. I. Three-dimensional structure of the armadillo repeat region of beta-catenin. Cell 90:871-882, 1997.

10. Huber, A. H., and Weis, W. I. The structure of the beta-catenin/E-cadherin complex and the molecular basis of diverse ligand recognition by beta-catenin. Cell 105:391-402, 2011. 
11. Ishijima, A., Kojima, H., Higuchi, H., Harada, Y., Funatsu, T., and Yanagida, T. Multipleand single-molecule analysis of the actomyosin motor by nanometer-piconewton manipulation with a microneedle: unitary steps and forces. Biophysical journal 70:383400, 1996.

12. Jaalouk, D. E., and Lammerding, J. Mechanotransduction gone awry. Nature reviews. Molecular cell biology 10:63-73, 2009.

13. Kobielak, A., and Fuchs, E. Alpha-catenin: at the junction of intercellular adhesion and actin dynamics. Nature reviews. Molecular cell biology 5:614-625, 2004.

14. Law, R., Liao, G., Harper, S., Yang, G., Speicher, D. W., and Discher, D. E. Pathway Shifts and Thermal Softening in Temperature-Coupled Forced Unfolding of Spectrin Domains. Biophysical Journal 85: 3286-3293, 2003

15. Lecuit, T. $\alpha$-catenin mechanosensing for adherens junctions. Nature Publishing Group 12:522-524, 2010.

16. Lee, G., Abdi, K., Jiang, Y., Michaely, P., Bennett, V., and Marszalek, P. E. Nanospring behaviour of ankyrin repeats. Nature 440:246-249, 2006.

17. Popa, I., Kosuri, P., Alegre-Cebollada, J., Garcia-Manyes, S., and Fernandez J. M. Force dependency of biochemical reactions measured by single-molecule force-clamp spectroscopy. Nature Protocols 8:1261-1276, 2013

18. Madler, S., Bich, C., Touboul, D., and Zenobi, R. Chemical cross-linking with NHS esters : a systematic study on amino acid reactivities. Journal of mass spectrometry 44 : 694-706, 2009.

19. Madox, H. P., and Jenkins, D. 3-Aminopropyltriethoxysilane (APES): a new advance in section adhesion, tissue patterns and morphogenesis, Journal of clinical pathology, 40: 1256-1260, 1987.

20. Marko, J. F., and Siggia, E. D. Stretching DNA. Macromolecules 28:8759-8770, 1995.

21. Miyake, Y., Inoue, N., Nishimura, K., Kinoshita, N., Hosoya, H., and Yonemura, S. Actomyosin tension is required for correct recruitment of adherens junction components and zonula occludens formation. Experimental cell research 312:1637-1650, 2006.

22. Odorico, M., Teulon, J.-M., Bessou, T., Vidaud, C., Bellanger, L., Chen, S. W., Quenmeneur, E., Parot, P., Pellequer, J.-L. Energy landscape of chelated uranyl: 
antibody interactions by dynamic force spectroscopy. Biophysical journal 93:645-654, 2007.

23. Pilot, F., and Lecuit, T. Compartmentalized morphogenesis in epithelia: from cell to tissue shape. Developmental dynamics : an official publication of the American Association of Anatomists 232:685-694, 2005.

24. Popa, I., Kosuri, P., Alegre-Cebollada, J., Garcia-Manyes, S., and Fernandez, J. M. Force dependency of biochemical reactions measured by single-molecule force-clamp spectroscopy. Nature protocols 8:1261-1276, 2013.

25. Rief, M., Gautel, M., Oesterhelt, F., Fernandez, J. M., and Gaub, H. E. Reversible unfolding of individual titin immunoglobulin domains by AFM. Science 276:1109-1112, 1997.

26. Ritco, -V. M., Ababou, A., and Horton, M. Molecular plasticity of $\beta$-catenin: New insights from single-molecule measurements and MD simulation. Protein Science 16:1984-1998, 2007.

27. Sekine, T., Ando, K., Machida, M., and Kanaoka, Y. Fluorescent thiol reagents : V. Microfluorometry of thiol compounds with a fluorescent-labeled maleimide. Analytical biochemistry 48:557-568, 1972.

28. Shook, D., and Keller, R. Mechanisms, mechanics and function of epithelialmesenchymal transitions in early development. Mechanisms of development 120:13511383, 2003.

29. Smith, S. B., Finzi, L., and Bustamante, C. Direct mechanical measurements of the elasticity of single DNA molecules by using magnetic beads. Science 258:1122-1126, 1992.

30. Haverkamp, R. G., Marshall, A. T., and Williams, M. A. K. Model for stretching elastic biopolymers which exhibit conformational transformations. Physical Review E statistical, nonlinear, and soft matter physics 95:021907, 2007

31. Tepass, U. Adherens junctions: new insight into assembly, modulation and function. BioEssays : news and reviews in molecular, cellular and developmental biology 24:690695, 2002. 
32. Thiery, J. P., Engl, W., Viasnoff, V., and Dufour, S. Biochemical and biophysical origins of cadherin selectivity and adhesion strength. Current opinion in cell biology 24:614619, 2012.

33. Valbuena, A., Vera, A. M., Oroz, J., Menéndez, M., and Carrión-Vázquez, M. Mechanical properties of $\beta$-catenin revealed by single-molecule experiments. Biophysical journal 103:1744-1752, 2012.

34. Verbelen, C., Gruber, H. J., and Dufre, Y. F. The NTA - His 6 bond is strong enough for AFM single-molecular recognition studies. Journal of molecular recognition 20:490 494, 2007.

35. Wozniak, M. A, and Chen, C. S. Mechanotransduction in development: a growing role for contractility. Nature reviews. Molecular cell biology 10:34-43, 2009.

36. Xing, Y., Takemaru, K.-I., Liu, J., Berndt, J. D., Zheng, J. J., Moon, R. T., and Xu, W. Crystal structure of a full-length beta-catenin. Structure 16:478-487, 2008.

37. Yao, M., Qiu, W., Liu, R., Efremov, A., Cong, P., Seddiki, R., Payre, M., Lim, C. T., Ladoux, B., Mège, R-M., and Yan, J. Force-dependent conformational switch of acatenin controls vinculin binding, Nature communications 5:1261-1276, 2014.

38. Yonemura, S., Wada, Y., Watanabe, T., Nagafuchi, A., and Shibata, M. alpha-Catenin as a tension transducer that induces adherens junction development. Nature cell biology 12:533-542, 2010. 
Figures

(a)

$\alpha$-Catenin and

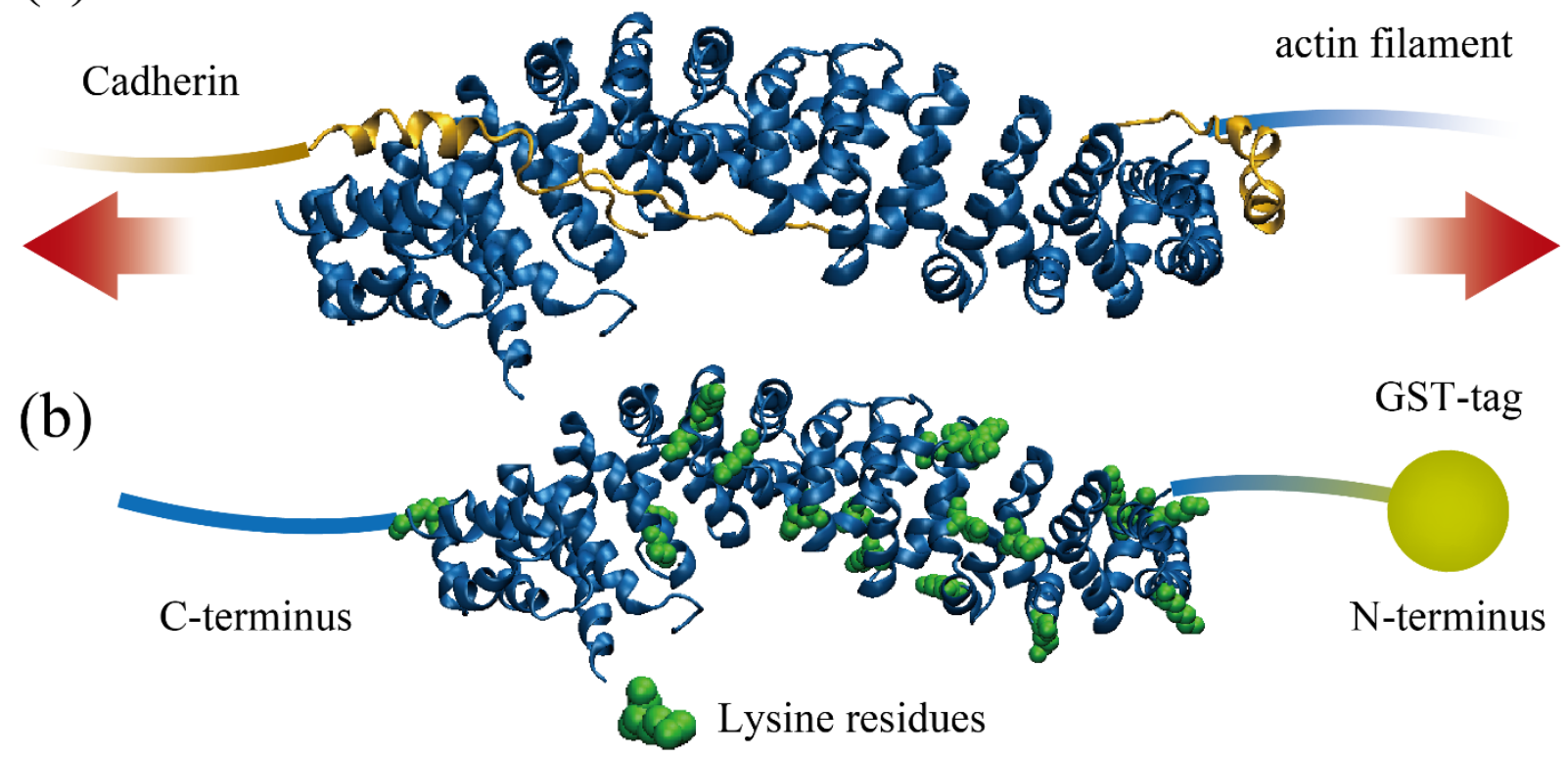

(c) His-tag

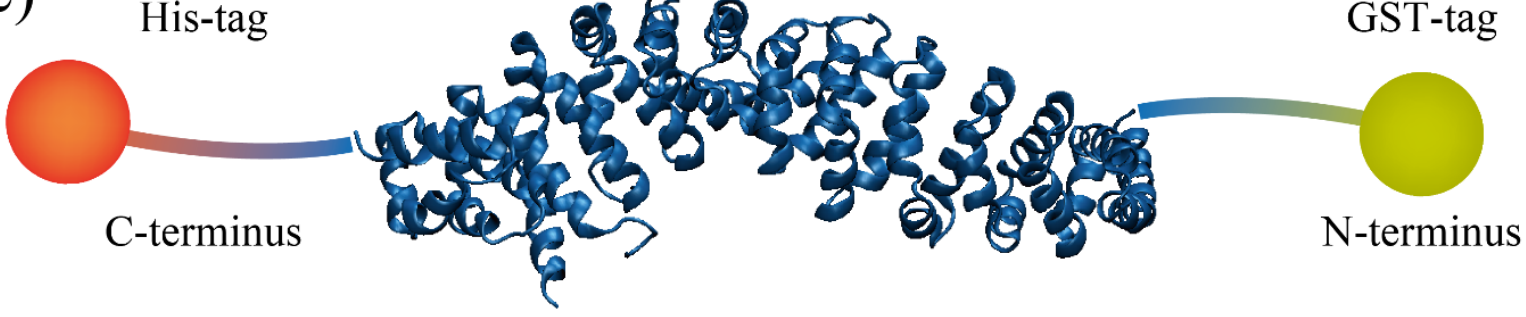

Figure 1: Schematic of $\beta$-catenin subjected to intercellular tension and $\beta$-catenin fusion

proteins. (a) The interaction condition between the cytoplasmic domain of cadherin and $\beta$ -

catenin. The $\mathrm{N}$-terminus of $\beta$-catenin directly interacts with $\alpha$-catenin and is exposed to

tension generated by the actomyosin contraction. (b) Single-tagged [N-terminus GST-tagged]

$\beta$-catenin with lysine residues (green) exposed to the solvent. The yellow sphere indicates the

GST tag. (c) Double-tagged [N-terminus glutathione-S-transferase (GST)-tagged and C-

terminus His-tagged] full-length $\beta$-catenin. The orange sphere indicates the His tag. 


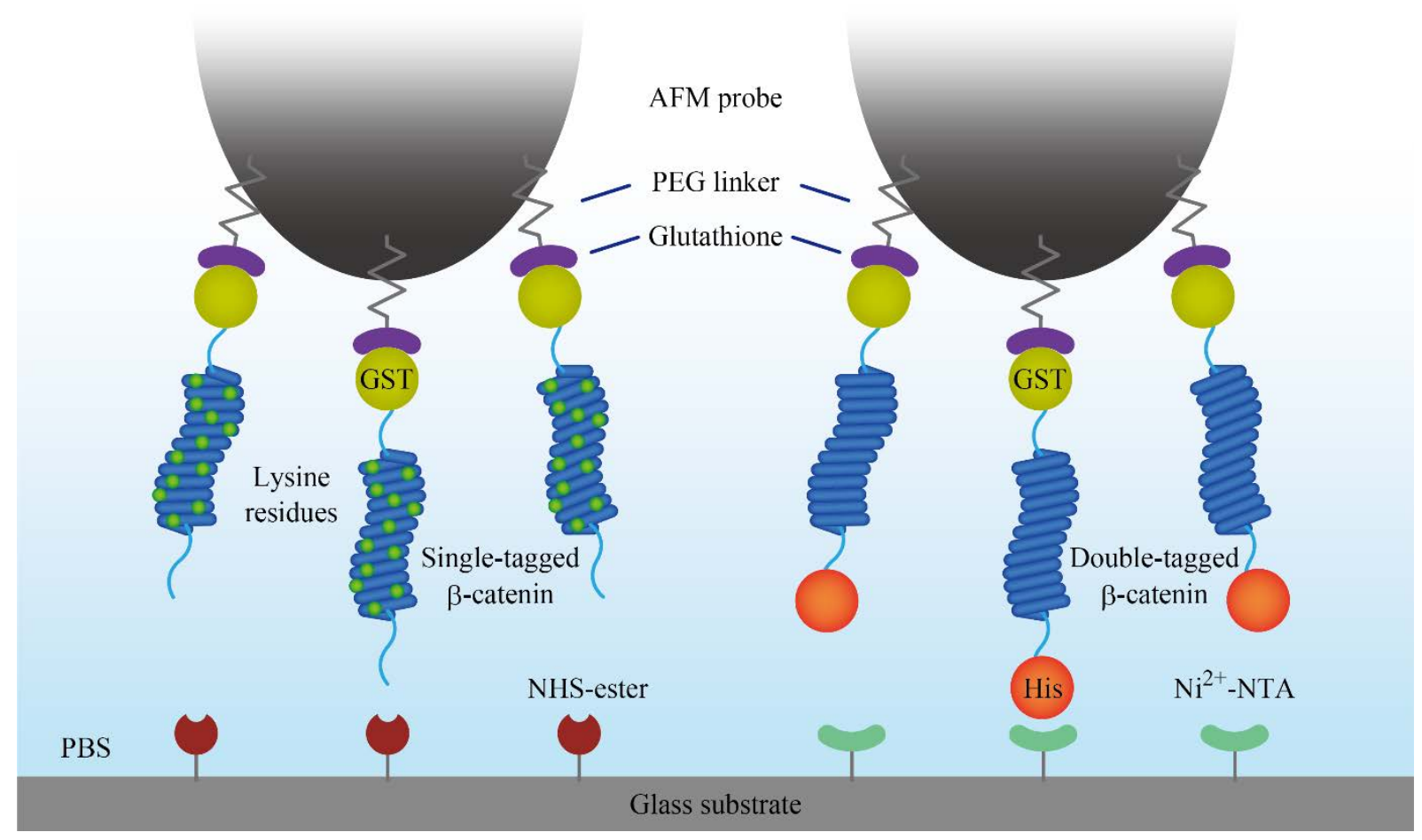

Figure 2: Schematics of AFM nanomechanical testing. Single-tagged (left) and double-tagged (right) $\beta$-catenin molecules modified on the AFM tip by the interaction between glutathione and the GST tag at the N-terminus. The AFM tip was then placed in contact with the substrate to allow interaction between 1) lysine residues and NHS-ester for single-tagged $\beta$-catenin and 2) the His tag at the C-terminus and $\mathrm{Ni}^{2+}$-NTA for double-tagged $\beta$-catenin. Finally, the AFM tip was moved upward and the $\beta$-catenin molecules were directly loaded. 

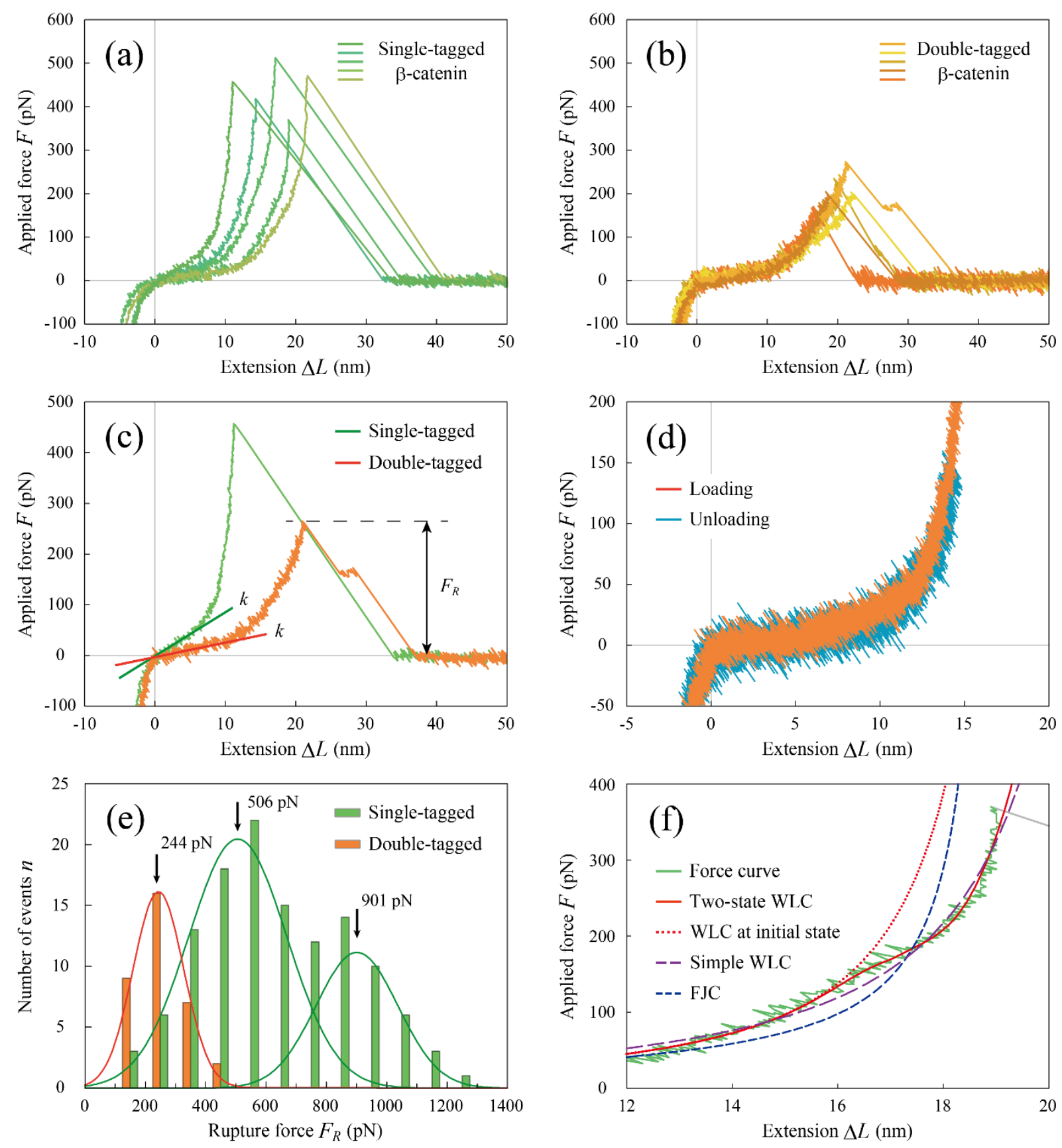

Figure 3: Results of the atomic force microscopy (AFM)-based spectroscopy of $\beta$-catenin.

(a,b) Force $(F)$ versus extension $(\Delta L)$ curves of the loading process obtained in single-tagged

(a, green line) and double-tagged (b, orange line) $\beta$-catenin experiments. (c) The rupture force

$\left(F_{\mathrm{R}}\right)$ was measured as the peak value of $F$ in the force curves. Spring constants $(k)$ in the

linear region were analyzed for single-tagged and double-tagged $\beta$-catenin. The orange and 
green linear lines indicate the linear regression curves in initial linear regions. (d) Force curve obtained with loading (orange) and unloading (blue) in double-tagged $\beta$-catenin experiments.

(e) Histogram of $F_{\mathrm{R}}$ from loading experiments using single-tagged (green) and double-tagged (orange) $\beta$-catenin with Gaussian curve fitting. (f) Results of curve fittings. Force curves (green line) were fitted by the two-state WLC model (red solid line), simple WLC model (purple long-dashed line), and FJC model (blue short-dashed line). The WLC curve at the initial state (red dotted line) was estimated from the result of fitting by the two-state WLC model. 